\title{
Impact of interaction between somatic illness and trait neuroticism on depressive symptoms
}

\author{
Krzysztof Malyszczak, MD, PhD* \\ Tomasz Wróbel, MD, PhD** \\ Angelika Chachaj, MD, PhD*** \\ Malgorzata Inglot, MD, PhD ${ }^{\star \star \star \star ~}$ \\ Andrzej Kiejna, MD, Prof*
}

* Department and Clinic of Psychiatry, Wroclaw Medical University

** Department and Clinic of Haematology, Blood Neoplasms, and Bone Marrow Transplantation, Wroclaw Medical University

*** Department and Clinic of Internal and Occupational Diseases and Hypertension, Wroclaw Medical University

**** Department and Clinic of Infectious Diseases, Hepatology and Acquired Immune Deficiencies, Wroclaw Medical University

POLAND

\begin{abstract}
Background and Objectives: An interaction between neuroticism and burden of illness supports depressive symptoms even at subclinical level. We have assessed its effect in groups of patients with different kind of somatic illness.

Methods: Depressive symptoms (SCAN 2.1) and a level of neuroticism (EPQ-R) were assessed in inpatients from 3 different hospital wards, namely the general internal, hematological and infectious wards, and in controls from the general population.

Results: A total of 184 adult subjects were examined (45 with haematological malignancies, 46 treated for other, non-malignant internal diseases, 48 with HCV infection before treatment and 45 healthy persons as control). Differences in mean neuroticism scores were not statistically significant (ANOVA, $F=1.44, \mathrm{p}=0.23$ ) whereas differences in mean depression scores were statistically significant (ANOVA, $\mathrm{F}=6.34, \mathrm{p}<0.001$ ). Results of ANCOVA for separate-slopes model analysis revealed a statistically significant level of interaction between groups and neuroticism in their influence on depression mean scores $(\mathrm{F}$ $=22.9, \mathrm{p}<0.001)$. The residual effect of the group variable was weak $(\mathrm{F}=0.54, \mathrm{p}=0.21)$.

Conclusions: The interaction is a significant factor related to depressive symptoms and can be used in estimating the extent of the psychological impact of a burden of illness.
\end{abstract}




\section{Introduction}

Neuroticism is defined as a domain of personality contrasting emotional stability with emotional instability and negative affectivity. Descriptions of neuroticism have been given by Eysenck and Eysenck, Costa and McCrae, and Cloninger ${ }^{1-3}$. Watson and Clark prefer the term negative affectivity over neuroticism in order to highlight several aspects of this domain, including negative mood and cognitions, as well as low self-esteem $^{4}$. Individuals with a high level of neuroticism have a tendency to be distressed and upset. They experience negative moods and affective and cognitive states such as nervousness, tension, worry, anger, scorn, guilt, a sense of rejection, negative self-image, dissatisfaction and to some extent, sadness. The disposition of the neurotic individual to negative affect is stable. High-neurotic individuals report consistently higher levels of negative affect than low-neurotic ones across time and independently of the situation even in non-distressing conditions. Negative affect is related to depression and symptoms of anxiety, and consequently to depressive and anxiety disorders 5 .

There are two approaches when it comes to defining the manner in which neuroticism and life adversity, in the form of stressful life events (SLE) or long term difficulties (LTD), have a combined effect in producing depression. The additive model assumes that the impact of life adversity on the risk of depression is independent of the level of neuroticism. In this model, life adversity and neuroticism independently correlate with the severity of depressive symptoms (and the risk of illness), so that their respective effects can be added up and subtracted (i.e. through an analysis of covariance). The additive model has been applied in many studies based on correlational analyses ${ }^{6-11}$.
The multiplicative model, on the other hand, assumes that the impact of adversity depends on the level of neuroticism. In this model, the level of neuroticism modifies the effect of life adversity on depressive symptoms by increasing susceptibility to depression and depressive symptoms. There is an increasing number of reports that neuroticism and SLE/LTD are independent risk factors for depression, interacting with each other ${ }^{12-14}$. Most of these studies focus on the relationship between neuroticism, SLE/LTD and diagnosis of depression, but diagnosis depends first and foremost on an exacerbated subthreshold process. Along with other factors, this process, based on the interaction between neuroticism and SLE/LTD, supports depressive symptoms and contributes to depressive episodes ${ }^{12,15}$. The aim of our study was to test a hypothesis that depressive symptoms, even subthreshold, are an effect of interaction between stressful situation caused by somatic illness and the level of neuroticism.

\section{Methods}

Depressive symptoms and a level of neuroticism were assessed in inpatients from 3 different hospital wards, namely the general internal, hematological and infectious wards, and in controls from the general population. We assumed that variations in the type of illness produced variations in the kind of LTD that in each case contributed to depressive symptoms. Interviews included a short screening test for possible psychotic states or dementia, followed by an assessment of depressive symptoms using a DSM-IV criteria scale based on the Schedules for Clinical Assessment in Neuropsychiatry (SCAN $2.1)^{16,17}$. Ratings of symptoms were used to 
calculate total severity of depressive state and to make a diagnosis of major depressive disorder. Neuroticism was measured using the 24-item scale from the Eysenck Personality Inventory - Revised (EPQ-R) ${ }^{1}$.

\section{Participants}

For the duration of the time set aside for assembling groups of subjects, all patients admitted to the selected wards were screened, and patients older than 18 whose psychiatric state allowed for examination (lack of psychotic state, disturbances of consciousness or dementia) were considered for inclusion in the study. Subjects were informed in detail about the interview and were asked for their consent to participate. After providing informed consent the subjects were interviewed and subsequently given questionnaires to complete. Subjects from the hematological ward were additionally screened in order to assemble a group with diagnoses of only hematological malignancies. Patients chosen from the infectious ward suffered from hepatitis $\mathrm{C}$ and were assessed just before beginning anti-HCV treatment. There was no additional selection procedure for patients from the general internal ward.

\section{Measurement}

Diagnoses of depression were based on the DSM-IV criteria using SCAN 2.0 questionnaires. The SCAN materials were translated into Polish at the Department of Psychiatry at Wroclaw Medical University as part of an international study EDEN (European Day Hospital Evaluation $)^{18}$. After translation a preliminary assessment of validity (in comparison with clinical diagnoses) and reliability was made ${ }^{19,20}$. Only sections relevant to depression were used; that is section 6 - depressed mood and ideation, section 7 - thinking, concentration, energy and interests, and section 8 bodily functions. Intensity of symptoms was rated on the standard 4-point (0-3) SCAN rating scale $\mathrm{I}$. The factors chiefly involved are intensity (intrusiveness and extent of interference with mental functions) and frequency of symptoms. The standard definitions apply to a period of about 4 to 6 weeks. Trained interviewers (two physicians and two medical students) assessed the presence and severity of symptoms based on information from patients, observation of affect, mood and behavior, and their own impressions. Diagnoses were made by applying the DSM-IV diagnostic criteria.

Separate ratings of depressive symptoms were added up in order to determine total severity of depression. This procedure set up a criteria scale for depression containing 9 items; total depression ratings ranging from 0 to 27. Personality trait neuroticism was assessed with the 24-item neuroticism scale from the Eysenck Personality Questionnaire - Revised (EPQ-R) in Polish adaptation by Brzozowski and Drwal ${ }^{21}$.

\section{Statistical analysis}

The effect of neuroticism on depressive symptoms at different levels of burden caused by physical illness was assessed using the ANCOVA (analysis of covariance) for separate-slopes model. In order to compensate for the possibility of a state effect (i.e. a rise in neuroticism ratings due to a state of depression) analysis of covariance was performed twice - once with real neuroticism ratings, and once with neuroticism ratings adjusted (reduced) in proportion to depression ratings. We assumed that the state effect occurs in the case of major de- 
pression with a coefficient of 0.2 for major depression. That means that a positive diagnosis of major depression implies a rise of $20 \%$ in neuroticism level (according to a study by Ormel et al.), and this rise is proportional to depression severity ${ }^{22}$. This can be calculated using the formula N_k $=\mathrm{N} /(1$ $+\mathrm{D} / 50$ ), where N_k is corrected neuroticism level, $\mathrm{N}$ is real neuroticism level and $\mathrm{D}$ is the patient's depression rating. The probit nonlinear model was used to assess the influence of neuroticism and kind of illness on diagnosis of depression. Calculations were done using Statistica for Windows 7.1 by StatSoft Inc. Tulsa, USA.

\section{Results}

A total of 184 subjects aged 20-80 were examined. Group 1 consisted of 45 patients with haematological malignancies (myeloma multiplex- 9, lymphoma- 14, acute leukaemia22), group 2 comprised 46 patients treated for other, non-malignant internal diseases (hypertension, circulatory failure, angina pectoris, coronary disease, phlebothrombosis, bronchopneumonia, chronic obturatory pulmonary disease (COPD), pneumoconiosis, chronic lung embolism, adult-type diabetes mellitus, hiperlipidaemia, nephrolithiasis, gastric ulcer desease, lead-poisoning, vibration disease and degenerative joint disease), group
3 consisted of 48 patients with HCV infection before treatment with interferon $\alpha$ and ribavirin, and group 4 was made up of 45 healthy persons as control. All patients were assessed at clinics of the Medical University in Wroclaw. The basic demographic parameters of the subjects are presented in table 1 .

Differences between groups in the percentage of men and women were not statistically significant. The most distinct difference was between groups 1 and 3 (chi-square = $0.18, p=0.67)$. Differences in age among the groups were statistically significant (ANOVA, F = 4.1, p = 0.008). Subjects in groups 1 and 2 had a higher mean age than in groups 3 and 4. Post-hoc comparisons revealed that only the difference between groups 2 and 3 reached statistical significance (post-hoc Turkey test, $\mathrm{p}=0.044$ ). To a slight degree, patients' age correlated with their neuroticism score $(\mathrm{r}=0.17, \mathrm{p}=0.019)$ and depression score $(\mathrm{r}=0.19, \mathrm{p}=0.012)$.

Table 2 shows neuroticism and depression mean scores. Differences in mean neuroticism scores were not statistically significant (ANOVA, $\mathrm{F}=1.44, \mathrm{p}=0.23$ ). After compensating for the effect of age the significance level $p$ even decreased (ANCOVA, $\mathrm{F}=1.00, \mathrm{p}=0.4)$. Because of different mean neuroticism scores in men and women, separate comparisons were carried out. Groups did not significantly differ in mean neuroticism score for men (ANOVA, $\mathrm{F}=0.75, \mathrm{p}=$ 0.52; ANCOVA, $\mathrm{F}=0.59, \mathrm{p}=0.63$ ), nor for

Table 1

Age and gender of subjects

\begin{tabular}{llccc} 
& Group & Total $n$ & \multicolumn{1}{c}{ Men/women } & Mean age (min-max) \\
\hline 1 & Haematologic malignancies & 45 & $25 / 20(55.6 \% / 44.4 \%)$ & $55.3(23-73)$ \\
\hline 2 & Internal diseases & 46 & $24 / 22(52.2 \% / 47.8 \%)$ & $55.9(21-88)$ \\
\hline 3 & HCV & 48 & $25 / 23(52 \% / 48 \%)$ & $48.5(22-65)$ \\
\hline 4 & Healthy & 45 & $23 / 22(51.1 \% / 49.9 \%)$ & $48.8(20-82)$ \\
\hline
\end{tabular}


Table 2

Mean depressive symptoms and neuroticism scores; prevalence of MDD

\begin{tabular}{|c|c|c|c|c|c|}
\hline Group & $\begin{array}{c}\text { Mean } \\
\text { neuroticism } \\
\text { score } \pm \mathrm{SE}\end{array}$ & $\begin{array}{c}\text { Mean } \\
\text { neuroticism } \\
\text { score } \pm \text { SE } \\
\text { men/women }\end{array}$ & $\begin{array}{c}\text { Mean } \\
\text { depression } \\
\text { score } \pm \text { SE }\end{array}$ & $\begin{array}{c}\text { Mean } \\
\text { depression } \\
\text { score } \pm \text { SE } \\
\text { men/women }\end{array}$ & $\begin{array}{c}\text { Diagnoses } \\
\text { of } \\
\text { depression }\end{array}$ \\
\hline \multirow{2}{*}{$\begin{array}{l}\text { Haematologic } \\
\text { malignancies }\end{array}$} & \multirow{2}{*}{$11.3 \pm 0.88$} & $10.6 \pm 1.17$ & \multirow{2}{*}{$6.33 \pm 0.72$} & $6.12 \pm 1.00$ & \multirow{2}{*}{$13(28.9 \%)$} \\
\hline & & $12.1 \pm 1.28$ & & $6.60 \pm 1.02$ & \\
\hline \multirow[t]{2}{*}{ Internal diseases } & \multirow{2}{*}{$12.6 \pm 0.87$} & $10.2 \pm 1.30$ & \multirow{2}{*}{$4.57 \pm 0.71$} & $4.00 \pm 1.12$ & \multirow{2}{*}{$4(8.7 \%)$} \\
\hline & & $14.5 \pm 1.12$ & & $5.00 \pm 0.90$ & \\
\hline \multirow[t]{2}{*}{$\mathrm{HCV}$} & \multirow{2}{*}{$11.3 \pm 0.85$} & $10.0 \pm 1.17$ & \multirow{2}{*}{$3.93 \pm 0.72$} & $3.50 \pm 1.07$ & \multirow[b]{2}{*}{$7(15.2 \%)$} \\
\hline & & $12.6 \pm 1.19$ & & $4.35 \pm 0.95$ & \\
\hline \multirow[t]{2}{*}{ Healthy } & \multirow{2}{*}{$10.0 \pm 0.88$} & $8.3 \pm 1.22$ & \multirow{2}{*}{$1.93 \pm 0.72$} & $1.52 \pm 1.04$ & \multirow{2}{*}{$1(2.2 \%)$} \\
\hline & & $11.9 \pm 1.22$ & & $2.36 \pm 0.97$ & \\
\hline
\end{tabular}

$\mathrm{SE}$ - standard error.

Table 3

Regression and correlation coefficients between neuroticism (N) and depressive symptoms severity (D)

Neuroticism score

Adjusted neuroticism score

\begin{tabular}{lcccc}
\cline { 2 - 5 } Group & Regression $\mathrm{D}=$ & $\begin{array}{c}\text { Correlation between } \\
\mathrm{D} \text { and } \mathrm{N}\end{array}$ & Regression $\mathrm{D}=$ & $\begin{array}{c}\text { Correlation between } \\
\mathrm{D} \text { and N }\end{array}$ \\
\hline $\begin{array}{l}\text { Haematologic } \\
\text { malignancies }\end{array}$ & $-6.37+1.54 * \mathrm{~N}$ & 0.577 & $-2.46+1.37 * \mathrm{~N}$ & 0.410 \\
\hline Internal diseases & $-4.18+0.84 * \mathrm{~N}$ & 0.541 & $-2.05+0.74 * \mathrm{~N}$ & 0.411 \\
\hline $\mathrm{HCV}$ & $-4.39+1.04 * \mathrm{~N}$ & 0.724 & $-4.23+1.14 * \mathrm{~N}$ & 0.660 \\
\hline Healthy & $3.63+0.17 * \mathrm{~N}$ & $0.149^{1}$ & $4.04+0.13 * \mathrm{~N}$ & $0.112^{1}$
\end{tabular}

${ }^{1}$ Statistically not significant.

women (ANOVA, $\mathrm{F}=1.06, \mathrm{p}=0.37$; ANCOVA, $F=0.72, p=0.55)$. Age had the same insignificant effect on neuroticism in all the groups $(\mathrm{F}=0.4, \mathrm{p}=0.76)$.

Differences in mean depression scores were statistically significant (table 2), even after making allowances for age (ANOVA, $\mathrm{F}=6.34, \mathrm{p}<0.001 ;$ ANCOVA, $\mathrm{F}=5.27, \mathrm{p}=$ $0.002)$. Groups differed significantly with respect to mean depression score for men (ANOVA, $\mathrm{F}=3.46, \mathrm{p}=0.02$; ANCOVA, $\mathrm{F}=$ $3.36, \mathrm{p}=0.023$ ) and for women (ANOVA, $\mathrm{F}$
$=2.86, \mathrm{p}=0.042$ ), but after compensating for the effect of age, differences decreased to just under the level of statistical significance ANCOVA, $\mathrm{F}=2.65, \mathrm{p}=0.054)$. Age had the same effect on depression in all the groups $(\mathrm{F}=0.45, \mathrm{p}=0.72)$.

There was a high degree of correlation between depression scores and neuroticism scores (figure 1 and table 3 ). The main aim of the analyses was to assess the influence of neuroticism on depression scores in groups. The ANCOVA method requires that 
the continuous covariate (neuroticism in this case) has the same effects on the dependent variable (i.e. depression) at different levels of the independent factor (i.e. the groups). As can be seen in table 3 and fig. 1, the slopes of regression lines vary among the groups. An analysis of the homogeneity of the slopes revealed a high level of interaction between the group and neuroticism variables $(\mathrm{F}=7.16, \mathrm{p}<0.001)$, so the $\mathrm{AN}$ COVA for separate-slopes model was used. The results revealed a statistically significant level of interaction between groups and neuroticism in their influence on depression mean scores $(\mathrm{F}=22.9, \mathrm{p}<0.001)$. The residual effect of the group variable was weak $(F=0.54, p=0.21)$. The same calcu- lations performed on adjusted neuroticism levels revealed slightly lower, but still statistically significant associations between depression ratings and group*neuroticism interaction: no homogeneity of slopes $(\mathrm{F}=3.77$, $\mathrm{p}$ $<0.012$ ), interaction between neuroticism and group $(\mathrm{F}=9.30, \mathrm{p}<0.001)$, and a weak, insignificant residual effect of the group $(\mathrm{F}=$ $0.97, p=0.41$ ). Thus, variations in depression scores among groups resulted mainly from the interaction between the group and neuroticism variables. The measure of this interaction is coefficient a of a linear regression equation $(\mathrm{aX}+\mathrm{b})$. This effect is showed in fig. 1. An analysis of diagnoses of depression using a probit model yielded no clear results. The full factorial method only revealed the
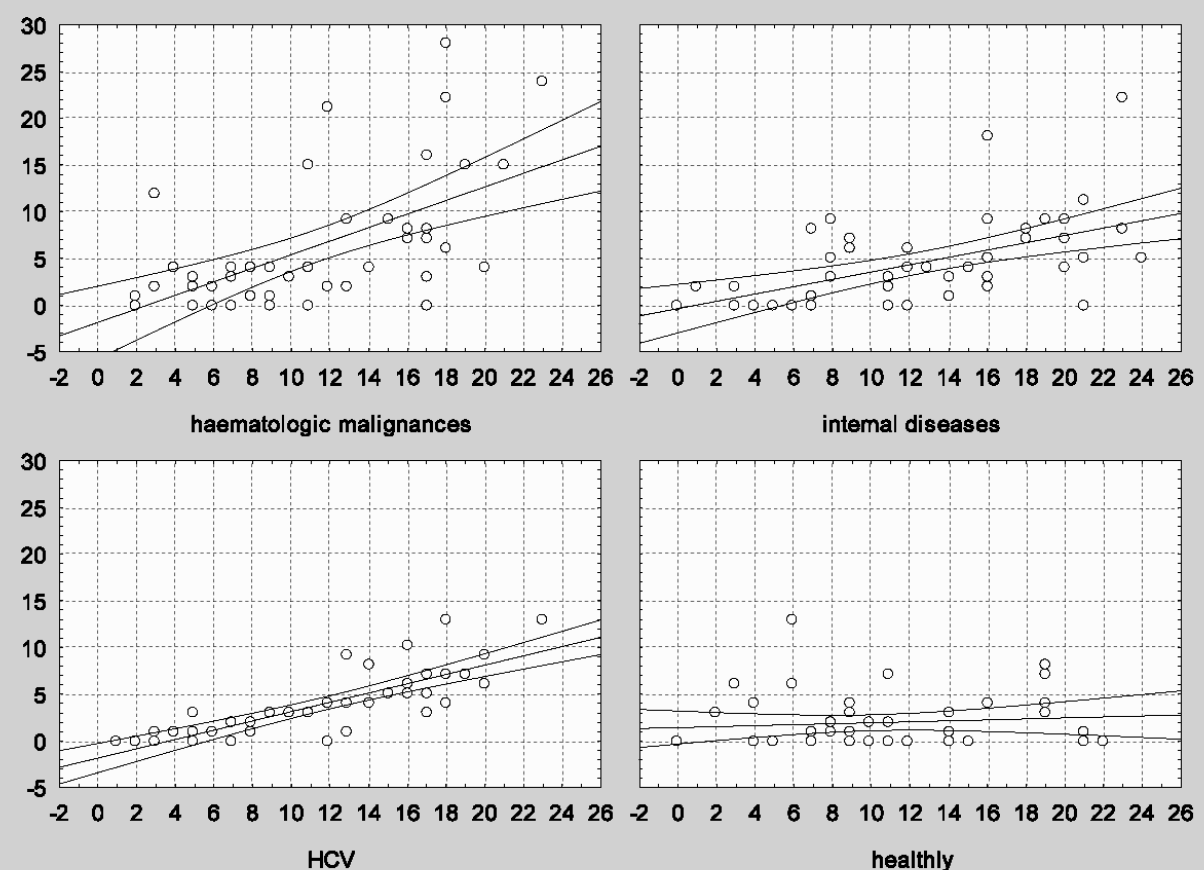

Neuroticism

Figure 1. Depressive symptoms vs neuroticism; scatterplots and regression lines; the steeper slope for haematologic malignances presents the stronger effect of interaction between kind of illness and neuroticism.

Horizontal line for healthy subjects presents no effect of interaction. 
significant effect of the intercept. Separate analyses confirmed the significant effect of the group variable (Wald stat. $=11.29, \mathrm{p}=$ 0.01 ), and neuroticism*group interaction (Wald stat. $=18.7, \mathrm{p}=0.0003$ ) with the prominent effect of the intercept.

\section{Discussion}

It is difficult to compare the burden of a disease (stress) in the respective groups, but we can assume that it is highest in the group with haemathologic malignancies, lower in groups with internal diseases and $\mathrm{HCV}$, and lowest in the control group. It can be assumed that psychological stress due to an illness is the main inter-group factor influencing the severity of depressive symptoms. Other potential factors are medication and the psychological effect of a bad somatic state, especially in groups with haemathologic malignancies. These two factors are only weakly related to patients' neuroticism score, and it is unclear whether they interact with neuroticism. The results of ANCOVA analysis reveal the interaction between the neuroticism and kind of illness to be a strong predictor of the severity of depressive symptoms, and far more prominent than the effect of the illness not linked to neuroticism. Differences in mean depressive score among groups can be seen as the consequence of variations in slopes of regression lines between neuroticism and depression. The regression line is almost horizontal in the control group. Its slope rises along with mean depressive score and is the highest in the haemathologic group. So, the slope of the regression line reflects the strain of depressiogenic factors caused by diseases and treatment typical for each group. The reverse of this conclusion can be also seen: stressful life events interact with neuroticism levels in the development of depressive symptoms, so symptoms severity is a product of these two factors. It is not merely an obvious idea, but in fact also a mathematical equation.

This relationship was established at diagnosis level in several studies. Kendler et al. evaluated three potent risk factors for major depresion: the personality trait of neuroticism, sex, and psychosocial adversity, operationalized by measures of recent stressful life events (SLE) ${ }^{14}$. Their results revealed an interaction between neuroticism and adversity such that individuals with high neuroticism were more sensitive to the depressogenic effects of adversity. The influence of this interaction is substantial: the hazard ratio for the onset of major depression as result of severe SLE in individuals with low neuroticism was 8-9, whereas in individuals with high neuroticism, it was above 30 . Conversely, in the case of a lack of SLE, the hazard ratio in individuals with high neuroticism ranged from about 2.5 (men) to 5 (women). The relationship between neuroticism and adversity becomes directly multiplicative, except at high levels of long-term contextual threat, when it was slightly less than multiplicative. Ormel et al. evaluated neuroticism, SLEs and long term life difficulties as risk factors of depression in later life ${ }^{12}$. They found multiplicative interaction between long term difficulties and neuroticism, but not SLEs, to be a composite risk factor for major as well as subsyndromal depression, and suggested the hypothesis of etiological continuity between major depression and subsyndromal states. From a clinical point of view, major depression causes greater concern than subsyndromal depression, but from an etiologic point of view the difference between syndromal and subsyndromal states is only qualitative. The 
results of our study demonstrate that the stress-vulnerability model can be applied at symptomatic level. The linear regression of depressive symptoms/neuroticism slopes varied statistically between groups, which means that the influence of neuroticism on depressive symptoms varies according to the severity of illnesses in groups. Stress caused by disease and its treatment influences the slope of the regression line, which ranges from nearly 0 in the control group to 0.83 in the group with haemathologic malignancies. The charts in fig. 1 show the relationship between neuroticism level, the severity of depressive symptoms and stress as expressed by the variables $\mathrm{Y}$ and $\mathrm{X}$, and a slope (coefficient a of the linear equation $\mathrm{Y}$ $=\mathrm{aX}+\mathrm{b}$ ) of linear regression. Two conclusions arise from this relationship: (1) the interaction between LTD and neuroticism is a significant etiological factor influencing depression and (2) the severity of depressive symptoms is proportional to both neuroticism and life adversity.

In our study it was not possible to measure neuroticism level before the onset of illness, so the relationship between neuroticism and depressive symptoms is enlarged by a state effect. Analysis of adjusted data showed that the regression line slope decreased in two groups, increased in one group, and remained the same (insignificant) in the control group (table 3). Compensating for the state effect seems not to nullify the effect of linear D = kind of illness*neuroticism interaction, but merely to decrease its impact. This is noticeable as a decrease of Person product moment correlation coefficients between symptoms severity and neuroticism. This correlation is a measure of the strength of interrelation between kind of illness*neuroticism interaction and symptoms. Because the calculation of linear regression refers to average values in groups, it also refers to the average value of stress. Intra-group calculations of correlation are not dependent on the average stress caused by illness, but are dependent on interpersonal differences in stress, which decrease product correlation coefficients. Because we have not assessed individual stressful events, we could not calculate the values of the correlation between interaction and symptoms more precisely. Rough calculations based on average stress values yield decreased product-moment correlation. The state effect has the opposite influence on correlation. Consequently, after compensating for state effect, correlation coefficients are lower, but still significant.

The results of probit-model analysis point out that diagnosis of depression is predominantly related to factors other than neuroticism and group (as shown by the predomination of the intercept, which is not related to these factors). Diagnosis depends on a more complicated structure than total symptoms severity, so individual differences in stress (due to occupational, family and social problems) and differences in the biological impact of an illness or medication could be highly significant. The relatively small number of subjects in this study admittedly created favourable conditions for the predomination of random factors; factor-diagnosis analysis needs hundreds rather than tens of individuals in each group. Nevertheless, the findings reveal clear relationships within the scope of total symptoms severity.

In addition to being of clinical importance, the interaction between LTD or SLE and neuroticism is important to the field of etiological research. Studies on the etiology of depression are difficult because of the changeability of depressive symptoms and their multifactorial origin. Perhaps there is no alternative to untangling a number different pathways leading to similar clinical 
states. Personality trait neuroticism forms one of these pathways, and genetic studies lead to an understanding of the origins of depression. It is worth mentioning that there are also many pathways unrelated to neuroticism, e.g. directly genetic, or through gene-environment interaction ${ }^{23-25}$. An example of such interaction is polymorphism in the serotonin (5-HT) transporter gene that not contributes significantly to human emotionality $^{26}$. In fact, unlike neuroticism measured with Eysenck's questionnaire, this kind of susceptibility to depression could not be related to egodystonic emotionality in the absence of SLE. Calculations of neuroticism*SLE interaction and consequent control of this variable allow analyses of risk factors for depression to be carried out on a diagnostic or symptomatic level.

\section{Limitations}

Instead of using a fixed scale of LTD we assumed that different kinds of illness cause different types of emotional stress. A consequence of this approach is the impossibility of controlling interpersonal differences in experienced stressful difficulties, which in turn leads to an underestimation of the effect of LTD*neuroticism interaction. The advantage of setting an illness as the LTD is that becoming ill is hardly correlated with neuroticism.

A cross-sectional outline of the study made it impossible to measure patients' neuroticism ratings before they became ill, so we were unable to control the state effect variable. The assumption that this effect is proportional to the severity of depressive symptoms arose out of observations of a scare effect - subthreshold depressive symptoms were seen to cause an increase in neuroticism level, but to a lesser degree than diagnosis of depression. Compensation for neuroticism ratings yield approximate pre-morbid values, but this is still only estimation and cannot replace pre-morbid assessment.

The relatively small number of examined subjects did not allow for an analysis of the prevalence of diagnosis of major depression: there was 1 case of major depression in the control group and 4 cases in the group with various internal diseases, which is insufficient data to reduce the effect of random factors by averaging.

\section{Acknowledgments}

Funding for this study was provided by Wroclaw Medical University (WMU) Grant No 909/03. The WMU had no further role in study design; in the collection, analysis, and interpretation of data; in writing of the report; and in the decision to submit the paper for publication.

There are no conflicts of interest for any of authors.

\section{References}

1. Eysenck HJ, Eysenck SBG. Manual of the Eysenck Personality Inventory. London: Hodder \& Stoughton; 1975.

2. Costa PT, McCrae RR. The five-factor model of personality and its relevance to personality disorders. J Pers Dis 1992; 6: 343-359.

3. Cloninger CR. A systematic method for clinical description and classification of personality variants. Arch Gen Psychiatry 1987; 44: 573-588.

4. Watson D, Clark LA. Negative Affectivity: The Disposition to Experience Aversive Emotional States. Psychol Bull 1984; 95: 465-490.

5. Clark LA, Watson D, Mineka S. Temperament, personality and the mood and anxiety disorders. J Abn Psychol 1994; 103: 103-116. 
6. Duggan CF, Lee AS, Murray RM. Does personality predict long-term outcome in depression? Br J Psychiatry 1990; 157: 19-24.

7. Barrio V, Moreno-Rosset C, López-Martinez R, Olmedo M. Anxiety, depression and personality structure. Pers Individ Diff 1997; 23: 327-335.

8. Wilhelm K, Parker G, Dewhurst-Savellis J, Asghari A. Psychological Predictors of single and recurrent major depressive episodes. J Affect Dis 1999; 54: 139-147.

9. Whittington JE, Huppert FA. Neuroticism, psychiatric symptoms and life events. Pers Individ Diff 1998; 24: 97-107.

10. Schmitz N, Kugler J, Rollnik J. On the relation between neuroticism, self-esteem, and depression: Results from the National Comorbidity Survey. Comp Psychiatry 2003; 44: 169-176.

11. Cox BJ, McWilliams LA, Enns MW, Clara IP. Broad and specific personality dimensions associated with major depression in a nationally representative sample. Compr Psychiatry 2004; 45: 246-253.

12. Ormel J, Oldenhinkel A, Brilman EI. The interplay and etiological continuity of neuroticism, difficulties, and life events in the etiology of major and subsyndromal, first and recurrent depressive episodes in later life. Am J Psychiatry $2001 ; 158: 885-892$.

13. Oldenhinkel A, Bouhuys AL, Brilman EI, Ormel J. Functional disability and neuroticism as predictors of latelife depression. Am J Geriatr Psychiatry 2001; 9: 241-248.

14. Kendler KS, Kuhn J, Prescott CA. The interrelationship of neuroticism, sex, and stressful life events in the prediction of episodes of major depression. Am J Psychiatry 2004; 161: 631-636.

15. Lyness JM, Duberstein PR, King DA, Cox C, Caine ED. Medical Illness Burden, Trait Neuroticism, and Depression in Older Primary Care Patients. Am J Psychiatry 1998; 155: 969-971.

16. Schedules for Clinical Assessment in Neuropsychiatry (SCAN) Version 2.1, Manual. Geneva: World Health Organization (WHO), Division of Mental Health; 1992.

17. Schedules for Clinical Assessment in Neuropsychiatry (SCAN) Version 2.1, Glossary. Geneva: World Health Organization (WHO), Division of Mental Health; 1992.

18. Kallert TW, Glockner M, Priebe S, Briscoe J, Rymaszewska J, Adamowski T, et al. A comparison of day hospitals in five European countries: implications of their diversity for day hospital research. Soc Psychiatry Psychiatr Epidemiol 2004; 39: 777-788.

19. Malyszczak K, Rymaszewska J, Hadryś T, Adamowski T, Kiejna A. Comparison between a SCAN diagnosis and a clinical diagnosis. Psychiatr Pol 2002; 36(6 Suppl.): 377-380.

20. Adamowski T, Kiejna A, Hadryś T. SCAN system semi-structured interview based on diagnostic criteria. Psychiatr Pol 2006; 40: 751-760.

21. Brzozowski P, Drwal RL. Kwestionariusz Osobowości Eysencka, polska adaptacja EPQ-R. Warsaw Poland: Pracownia Testów Psychologicznych PTP; 1995.

22. Ormel J, Oldehinkel AJ, Brilman EI, Nolen VA, Vollebergh W. Vulnerability before, during, and after a major depressive episode. A three-wave population-based study of state, scar and trait effects. Arch Gen Psychiatry 2004; 61: 387-392.

23. Kendler KS, Gardner CO, Prescott CA. Toward a comprehensive developmental model for major depression in men. Am J Psychiatry 2006; 163: 115-124.

24. Kendler KS, Gardner CO, Prescott CA. Toward a comprehensive developmental model for major depression in women. Am J Psychiatry 2002; 159: 1133-1145.

25. Caspi A, Sugden K, Moffitt TE, Taylor A, Craig IW, Harrington $\mathrm{H}$, et al. Influence of life stress on depression: moderation by a polymorphism in the 5-HTT gene. Science 2003; 301: 386-389.

26. Willis-Owen SA, Turri MG, Munafò MR, Surtees PG, Wainwright NW, Brixey RD, et al. The Serotonin Transporter Length Polymorphism, Neuroticism, and Depression: A Comprehensive Assessment of Association Biol Psychiatry 2005; 58: 451-456.

\footnotetext{
Address for correspondence:

Krzysztof Malyszczak, MD, PhD

Department and Clinic of Psychiatry,

Wroclaw Medical University

ul. L. Pasteura 10, 50-367 Wroclaw, Poland

Phone +48601750951

Fax +48717841602

E-mail: durlik@life.pl
} 